\title{
ASSESSMENT OF PERIPAPILLARY AND MACULAR VESSELS DENSITY ESTIMATED WITH OCT- ANGIOGRAPHY IN GLAUCOMA PATIENTS AND EFFECT OF INTRA OCULAR PRESSURE REDUCTION
}

\author{
Elsayed, S. ${ }^{1(*)}$, Mohamed, O. ${ }^{2}$ \& Fathy, A. ${ }^{2}$ \\ ${ }^{1}$ Ophthalmology dept., Faculty of Medicine, AL-Azhar Univ., Assuit, Egypt \\ ${ }^{2}$ Ophthalmology dept., Faculty of Medicine, Sohag Univ., Sohag, Egypt \\ *E-mail: drsarah.shandawily@gmail.com
}

\begin{abstract}
Purpose: Assessment of perpapillary and macular vessels density estimated with OCTAngiography in glaucoma patients and effect of intra ocular pressure reduction on them in 0,1 , 3, 6 months. Patients and methods: This is a prospective observational study that included 40 eyes of 35 patients with glaucoma (open, closed angles) at Sohag Investigation Center from April 2018 to April 2021. The study population underwent $4.5 \times 4.5 \mathrm{MM}$ scans centered on the optic nerve head \& $3 \times 3$ MM scans centered on the macula using Optical Coherence Tomography Angiography (OCTA) (RTVue XR Avanti Optovue, Fremont, CA) for measuring the perpapillary vascular density, RNFL thickness, GCL thickness and macular vascular density in glaucomatous eyes before and after treatment by 1, 3 months and 6 months. Results: Mean age of the study population was 42.6 years with SD 12.29 years and range from 30 to 63 years. There was a significant reduction of IOP from $(30.075 \pm 8.3939 \mathrm{mmHg})$ at baseline to maximum reduction at 6 months $(17.175 \pm 3.257 \mathrm{mmHg}, P=\mathbf{0 . 0 0 1})$. There was a non-significant increase in thickness peripapillary RNFL \& GCL from baseline to 6 months following IOP reduction $(P=0.479,0.234$ respectively). There was a slight significant increase in thickness of the superior, inferior, temporal, nasal peripapillary vascular density (more significant in superior and nasal quadrants) \& laminal vascular density (VD ID) from baseline to 6 months post treatment $(P=\mathbf{0 . 0 1}, \mathbf{0 . 0 1 9}$, $0.046,0.032,0.003)$ \& there was a significant increase of macular $V D$ in response to IOP reduction at 1, 3, 6 months $(P=\mathbf{0 . 0 5 4})$. There was found a strong significant correlation between all these items improvement with IOP reduction. Conclusion: IOP has a closed relation with macular and $\mathrm{ONH}$ vascularity that prove that vascular insult has a close relation of development or prognosis of glaucoma. OCTA is the new novel era that allows early diagnosis and good follow up for glaucoma patients.
\end{abstract}

Keywords: Glaucoma, ONH vascular density, Macular vascular density, IOP, Optical Coherence Tomography Angiography

\section{Introduction}

Glaucoma is the leading cause of irreversible blindness worldwide [1]. Since glaucomatous damage is preventable but irreversible, an early diagnosis and close follow-up of glaucoma patients are primordial [2]. Elevated intraocular pressure 
(IOP) is the leading risk factor for glaucoma [3]. It is thought that the cause of glaucoma has a "mechanical" compressive theory particularly in patients who have progressive disease despite low IOP levels \& for these patients and others; a "vascular" theory has been proposed that's impaired regulation of ocular blood flow results in periods of relative ischemia that damages the optic nerve [3]. Currently, there are 2 groups of complementary exams used for the diagnosis and follow-up of glaucoma patients: structural (where OCT has a considerable role) and functional (visual field) optic nerve measurements. Both technologies have strengths and limitations [2,4]. Although numerous technologies including fluorescein angiography (FA) [5], indo-cyanine green angiography (ICGA) [6], scanning laser ophthalmoscopy [7], laser Doppler flowmetry [8], and laser speckle flowgraphy [9] have been used to doc-ument the impairment of ocular blood flow and alterations of the retinal micro-vasculature in glaucoma, they also have limitations in determining the role

\section{Patients and Methods}

This is a prospective observational study that included 40 eyes of 35 patients (20 eyes were to females and 20 eyes were to males) who are diagnosed with glaucoma (open, closed angles) and get into treatment plan to control IOP. Mean age of patients in our study was 42.6 years with SD 12.29 years and range from 30 to 63 years, scans handheld at Sohag Investigation Center from April 2018 to April 2021. Written informed consent was obtained from each patient prior to their enrolment in the study.

Inclusion criteria: 1) Patients diagnosed with glaucoma in age above 30 years old. (High IOP in repeated visits, RNFL \& GCL lesions seen by OCT to optic disc and macula). 2) Patients with un treated glaucoma or high IOP not reaching target IOP. 3) Good quality scans of OCT \& OCTA. of the vascular dysregulation in glaucoma [10]. Optical coherence tomography angiography (OCTA) is a recently developed, noninvasive imaging modality that detects blood flow through the motion contrast generated by red blood cells. It can be used to provide a quantitative assessment of the microcirculation of the retina and choroid in various layers [11]. By the year 2017 the publications described that not only the papillary but also the macular VD is decreased in glaucomatous eyes as measured by OCTA [10], Shoji et al. showed that the mean rate of change in macular VD was significantly faster in glaucomatous eyes than in healthy eyes and that a change in VD could be detected before a change in ganglion cell complex (GCC) thickness occurs [10] that provides early diagnosis and follow up of glaucoma. This study aimed to assess peripapillary and macular vessels density estimated with OCT- Angiography in glaucoma patients and effect of intra ocular pressure reduction on them in $0,1,3,6$ months.

Exclusion criteria: 1) Patients with media opacities that interfere with imaging. 2) Patients with peripapillary atrophy that will give false vascular and nerve fiber data. 3) Patients with any vitreal or retinal diseases. 4) Patients that are un co-operative or with un regular visits. 5) Patients with non-responding to lowering IOP treatment. Images were excluded based on 1 or more of the following criteria: 1) Motions artifacts. 2) Poor quality of images. 3) Poor clarity obscuring view of vasculature. 4) Low signal strength index (SSI less than 47). 5) Segmentation errors. All patients underwent a comprehensive ophthalmic examination including slit lamp bio microscopy to exclude any media opacities and anterior segment lesions, measurement of IOP using Goldman applanation tonometer, gonioscopy using a 4 mirror non-indentation indirect gonioscope and stereo biomicroscopic examination of 
the optic nerve head using a 78D condensing lens. RNFL \& GCL thickness was measured using SD-OCT Avanti (RTVue XR Avanti System), OCT-A was performed

\subsection{OCT-A image acquisition and processing}

The Avanti SD-OCT device (RTVue XR Avanti System; Optovue Inc, Fremont, CA, USA) with AngioVue AngioAnalytic software was used for the study. It uses a Split-spectrum Amplitude Decorrelation Angiography (SSADA) algorithm to capture the dynamic motion of $\mathrm{RBCs}$ and generate a high-resolution three dimensional (3D) image of perfused retinal vasculature. OCT-A scans were acquired by trained and experienced technicians, AngioAnalytic scans in the current study consisted of: 3D Angio Disc 4.5x $4.5 \mathrm{~mm} \&$ 3D Angio Retina $3.0 \times 3.0 \mathrm{~mm}$.

\subsection{Follow up}

OCTA was used for measuring the peripapillary RNFL thickness, the $\mathrm{ONH}$ vascular density, macular GCL complex and macular vascular density before and reviewed after treatment of glaucoma

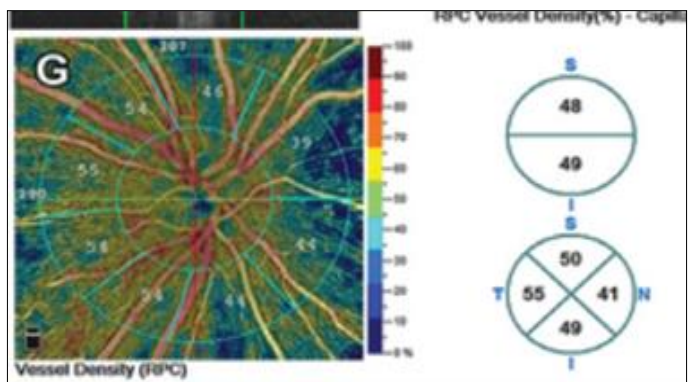

using the Avanti SD-OCT device (RTVue XR Avanti System; Optovue Inc, Fremont, CA, USA).
The software provides information on vessel density (VD), peripapillary RNFL \& GCL thickness. The superficial peripapillary region that is $700 \mathrm{um}$ from optic disc edge, Papillary region ( $3 \mathrm{~mm}$ circular region centered on the $\mathrm{ONH}$ ) that named as VD inside disc. The FAZ was automatically mapped by the software that extends to a diameter of $0.6 \mathrm{~mm}$ and parafovea is an annular region of 0.95 $\mathrm{mm}$ in width extending beyond the FAZ $\&$ the perifovea is an annular region of $1.5 \mathrm{~mm}$ in width circumscribing the parafovea.

and lowering of IOP by 1 month, 3 months and 6 months. We used the thickness map and vessel density map for follow up.

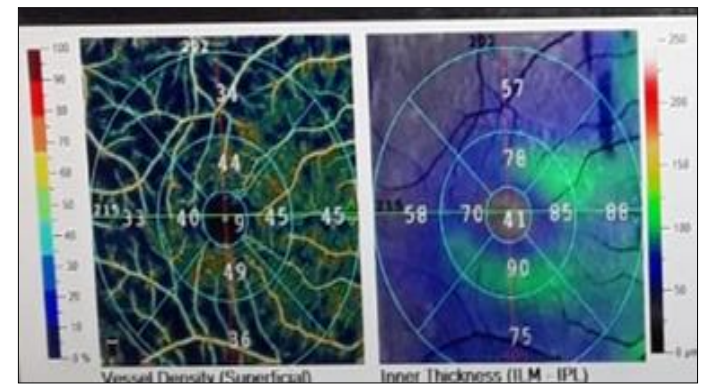

Figure 1: ONH \& macular maps in OCTA examination.

\subsection{Statistical analysis}

All statistical analyses were calculated using package for social sciences (IBM-SPSS), version 24 (IBM-Corporation, Chicago, USA; August 2017). P-value of $<0.05$ was considered statistically significant. Data expressed as mean, standard deviation (SD), number and percentage. Mean and standard deviation will be used as descriptive value for quantitative data. Student $t$ test used to compare the means between two groups, and oneway analysis of variance (ANOVA) test used to compare means of more than two groups; with LSD post HOC test to calculate the individual $\mathrm{p}$ values between each two groups. Mann-Whitney and Kruskal-Wallis tests used instead of student $t$ test and ANOVA for nonparametric data to compare medians rather than means between two or more groups; respectively. Paired t test used to compare the means in different times of follow up. Pearson Chi square test used to compare percentages of qualitative variables and Fisher's exact test used instead for non-parametric data. All sub- 
jects were informed of the goals and procedures in the study and written

\section{Results}

The study included a total of 40 eyes of 40 patients who experienced with glaucoma and underwent with treatment plan either medical (in 32 cases with eye drops: beta-blockers, Prostaglandins with or without carbonic anhydrase inhibitors) or surgical (in 8 cases: 4 eyes phaco- informed consent was obtained from all subjects before enrollment.

trabeculectomy, 3 eyes trabeculectomy with peripheral iridotomy, 1 eye deep sclerectomy). Age was ranged from 30 to 63 years. Table (1) demonstrates the demographic and clinical characteristics of the patients.

Table 1: Demographic and clinical data of the study.

\begin{tabular}{|l|c|}
\multicolumn{1}{c|}{ Characteristic } & Value \\
\hline Number of Eyes & 40 \\
\hline Age in Yrs (mean + SD) & $42.6 \pm 12.29$ \\
\hline Male/ Female & $20 / 20$ \\
\hline IOP & $30.075 \pm 8.3939$ \\
\hline Medical / Surgical TTT & $32 / 8$ \\
\hline Closed / Open angle & $12 / 28$ \\
\hline
\end{tabular}

\subsection{Changes in IOP}

IOP has highly significant change at 1 month from base line (30.075 \pm $8.3939)$ to $(24.950 \pm 4.749)$ from starting of treatment ( $\mathrm{P}$ value $<0.001)$, then continued its decrease with significant value

at 3 months from starting treatment plan $(20.056 \pm 3.567, \mathrm{P}$ value 0.02$)$ and at 6 months reaches its lower level with highly significant decrease from baseline value $(17.175 \pm 3.257, \mathrm{P}$ value $<0.001)$

\subsection{Changes in the thickness of the peripapillary RNFL \& GCL}

The average thickness of the peripapillary RNFL \& GCL in significantly

changed after 6 months of IOP reduction $(\mathrm{P}=0.479,0.234)$, tab. (2)

Table 2: Changes in RNFL, GCL thickness, peripapillary and superficial macular vessel density pre- and post-glaucoma treatment.

\begin{tabular}{|c|c|c|c|c|c|}
\hline \multirow{2}{*}{\multicolumn{2}{|c|}{ Baseline }} & \multicolumn{3}{|c|}{ Post TTT } & \multirow{2}{*}{ P value } \\
\hline & & 1 month & 3 months & 6 months & \\
\hline IOP & $30.075 \pm 8.39$ & $24.950 \pm 4.749$ & $20.056 \pm 3.567$ & $17.175 \pm 3.257$ & $<0.001$ \\
\hline Avg. RNFL & $76.000 \pm 6.5$ & $78.800 \pm 10.27$ & $82.500 \pm 8.768$ & $88.225 \pm 15.83$ & 0.479 \\
\hline Avg. GCL & $75.000 \pm 6.7$ & $78.200 \pm 9.009$ & $81.000 \pm 8.97$ & $86.575 \pm 13.78$ & 0.234 \\
\hline \multicolumn{6}{|c|}{ VD, \% (mean, 95\% CI) } \\
\hline $\begin{array}{l}\text { Peripapillary } \\
\text { - Avg. RPCD } \\
\text { - Superior } \\
\text { - Inferior } \\
\text { - Temporal } \\
\text { - Nasal } \\
\text { - Laminal ID } \\
\end{array}$ & $\begin{array}{c}36.200 \pm 8.10 \\
32.200 \pm 11.98 \\
32.200 \pm 11 . \\
39.200 \pm 8.595 \\
32.400 \pm 7.069 \\
44.400 \pm 1.919\end{array}$ & $\begin{array}{c}38.875 \pm 8.42 \\
34.050 \pm 11.29 \\
42.575 \pm 5.751 \\
40.325 \pm 6.044 \\
33.875 \pm 8.422 \\
46.725 \pm 6.008\end{array}$ & $\begin{array}{c}41.064 \pm 8.45 \\
37.200 \pm 10.65 \\
43.500 \pm 4.78 \\
40.850 \pm 5.98 \\
35.760 \pm 7.59 \\
47.450 \pm 5.98 \\
\end{array}$ & $\begin{array}{c}46.78 \pm 6.080 \\
45.44 \pm 9.950 \\
49.88 \pm 5.81 \\
42.100 \pm 12.48 \\
46.97 \pm 8.97 \\
50.200 \pm 4.608\end{array}$ & $\begin{array}{c}0.04 \\
0.01 \\
0.019 \\
0.046 \\
0.032 \\
0.003 \\
\end{array}$ \\
\hline $\begin{array}{l}\text { Macular } \\
\text { - Fovea } \\
\text { - Parafoveal } \\
\text { - Perifoveal } \\
\text { - Avg. macula }\end{array}$ & $\begin{array}{c}10.00 \pm 2.5981 \\
39.000 \pm 3.7749 \\
35.000 \pm 2.5981 \\
34.000 \pm 3.1225\end{array}$ & $\begin{array}{c}11.00 \pm 4.5826 \\
42.000 \pm 6.0622 \\
36.333 \pm 4.3589 \\
35.667 \pm 5.2915\end{array}$ & $\begin{array}{l}13.000 \pm 3.56 \\
44.560 \pm 6.55 \\
41.465 \pm 4.23 \\
38.000 \pm 5.19 \\
\end{array}$ & $\begin{array}{r}15.433 \pm 8.2574 \\
49.133 \pm 7.6010 \\
47.833 \pm 9.5234 \\
44.733 \pm 7.6200\end{array}$ & $\begin{array}{l}\mathbf{0 . 0 3 3} \\
0.081 \\
\mathbf{0 . 0 4 7} \\
\mathbf{0 . 0 5 4}\end{array}$ \\
\hline
\end{tabular}




\subsection{Changes in $\mathrm{ONH}$ vascular density}

The average superficial peripapillary vessel density RPCD changed slightly significantly after 6 months $(\mathrm{P}=$ 0.04) while deep laminal $\mathrm{ONH}$ vessel density showed the same manner that increased gradually with lowering IOP that increased at $1 \& 3$ months but was in significant and then increased significantly

at 6 months from baseline $(44.400 \pm$ 1.9198 to $50.200 \pm 4.608, \mathrm{P}=0.003$ ), fig. (2). By evaluating quadrant by quadrant, all quadrants showed at each follow up an improvement in their values by 6 months especially the superior and inferior quadrants ( $\mathrm{P}=0.01,0.019)$, fig. (3).

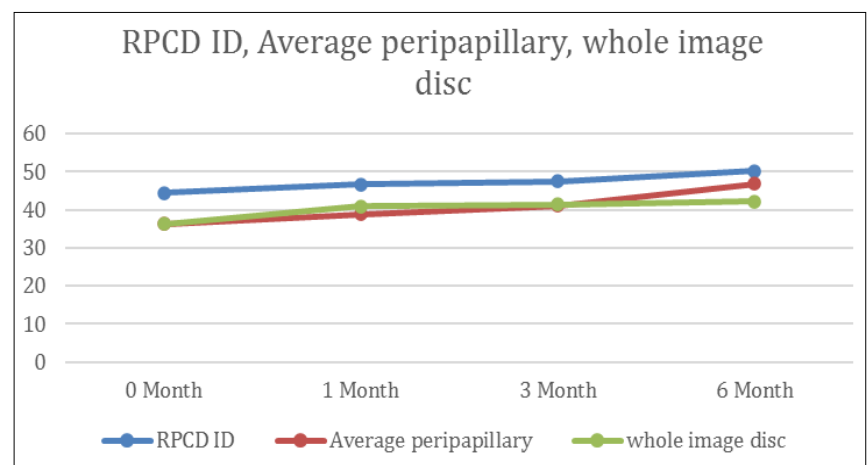

Figure 2: Changes in Average RPCD, RPCD ID, and Whole image disc.

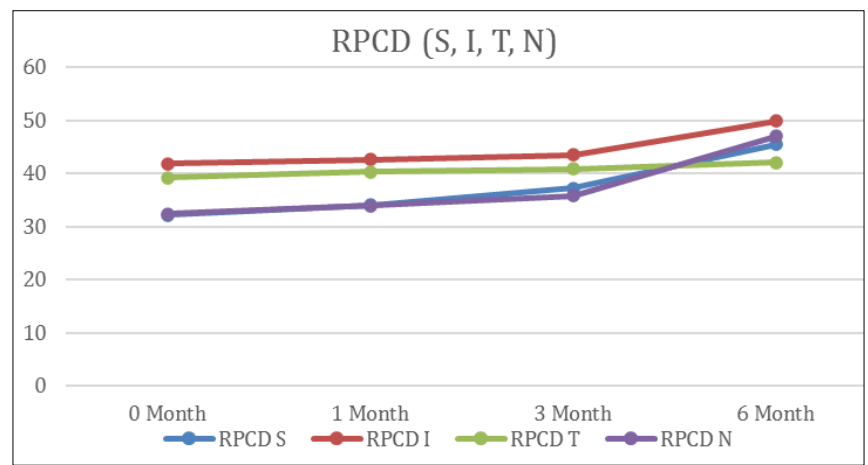

Figure 3: Changes in quadrants of Radial peripapillary capillary vascular density (RPCD).

\subsection{Changes in macular vascular density}

Average macular VD significantly increased at $1,3 \& 6$ months compared to baseline ( $34.000 \pm 3.1225$ to $44.733 \pm$ 7.6200 at 6 months, $P=0.054$ ). By detailed examination to macular areas fovea nonsignificant increased at 1 month but sign- ificant increased at 3,6 months. However, VD parafoveal significant increased at 1,3 months but at 6 months, there was non-significant difference to baseline. Furthermore, VD perifoveal significant increased at 1, 3, 6 months, fig. (4).

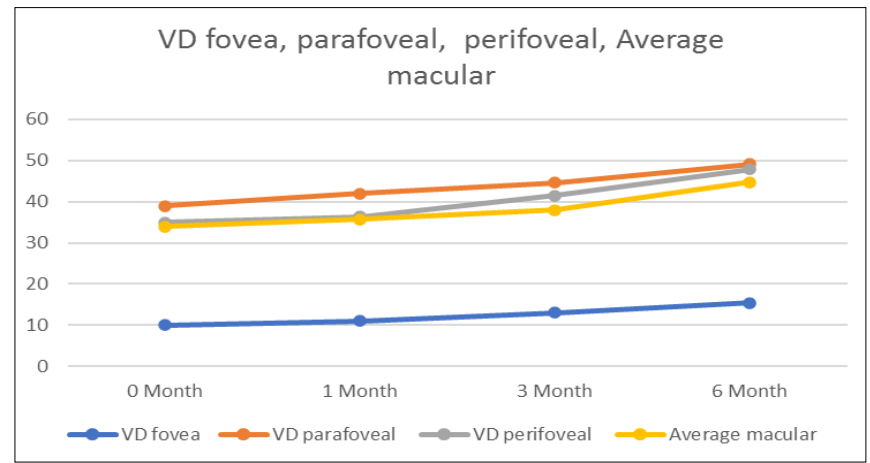

Figure 4: Changes in Foveal VD, Parafoveal VD, Perifoveal VD and Average macular VD. 


\subsection{Correlation between IOP and retinal VDs}

We found that at 6 month follow up, there was strong significant correlation between IOP and each of average macu-

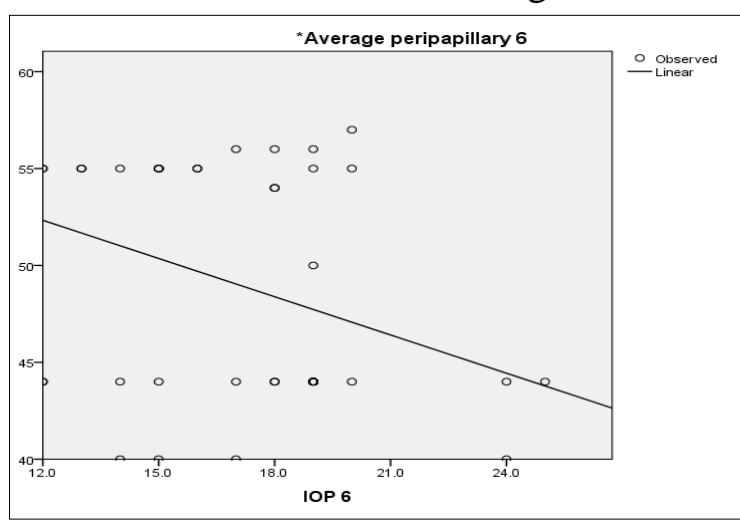

Figure 5: Correlation between IOP and average RPCD. lar VD and average peripapillary VD "AVG. RPCD", as could be seen in tab. (3) \& fig f $(5 \& 6)$.

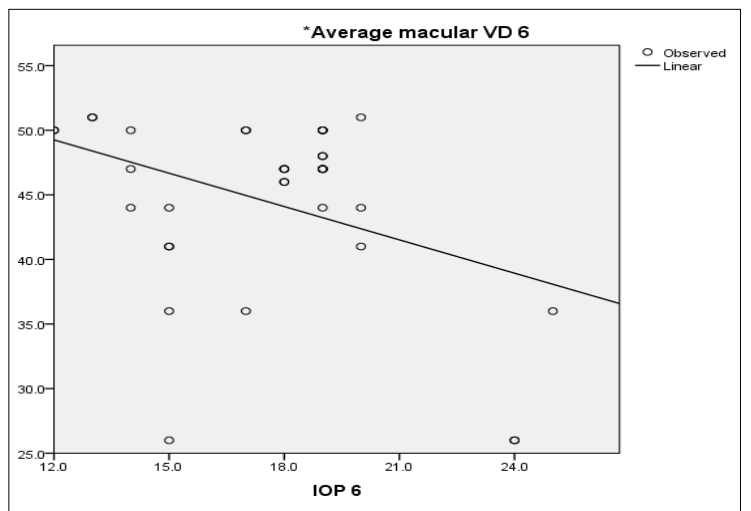

Figure 6: Correlation between IOP and average macular vessel density

Table 3: Correlation between IOP, Average macular VD, AVG. RNFL, AVG. GCL and Average peripapillary at 6 months.

\begin{tabular}{|l|l|c|c|c|c|c|}
\hline \multicolumn{2}{|c|}{} & IOP & $\begin{array}{c}\text { *Average } \\
\text { macular VD }\end{array}$ & AVG. RNFL & \multicolumn{2}{|c|}{ AVG. GCL $\begin{array}{c}\text { *Average } \\
\text { peripapillary }\end{array}$} \\
\hline \multirow{2}{*}{ IOP } & Pearson Correlation & 1 & $-.833-*$ & $-.648-*$ & $-.718-^{* * *}$ & $-.809-{ }^{* *}$ \\
\cline { 2 - 7 } & P value & & $<0.001$ & $<0.001$ & $<0.001$ & $<0.001$ \\
\hline
\end{tabular}

** Correlation is significant at the 0.01 level (2-tailed).

\section{Discussion}

There is evidence that retinal perfusion is auto-regulated in humans [12]. Impaired autoregulation may be a critical factor in several vascular diseases including glaucoma [13]. Liu et al. demonstrated higher repeatability and reproducibility of OCT angiography compared with other noninvasive techniques, such as laser Doppler flowmetry and laser speckle flowgraphy that have specific limitations, several studies have suggested that vascular factors can play a pathogenic role in glaucoma and decreased peripapillary and macular retinal vasculature identified by OCTA is clinically useful to observe the glaucoma progression [14-16]. It is now of interest to consider what happens after reduction of VD by pressure relief, whether by medical or surgical means. Our results suggest that reducing the IOP increases the ocular perfusion pressure, which enhances ocular blood flow that may contribute to the rate of glaucoma progression. In the present study, the management of IOP was achieved by using different therapeutic strategies, the mean IOP was $30.075 \pm$ $8.3939 \mathrm{mmHg}$ in the glaucomatous patients at baseline \& a significant IOP reduction, with an average value of $17.175 \pm 3.257 \mathrm{mmHg}(\mathrm{P}$ value $<0.001)$ at six months. Our results showed that by 6 months follow up there was a nonsignificant increase in RNFL \& GCL thickness. Near results found with de Paula et al. [17] they found that there is a significant increase of RNFL thickness 1 month post glaucoma treatment \& Sarkar et al. [18] described an RNFL thickening in each quadrant after trabeculectomy, which was significantly correlated with the magnitude of the IOP reduction. This finding may be explained with a reduction of the IOP compressive effect rather than a hypothetic neural regeneration. On the other hand, T. W. 
Ch'ng et al. [19] found that there is a transient increase in RNFL thickness 1 month after glaucoma surgery then has gradual decrease in thickness till 12 months follow up post-surgical, IOP at baseline was $19.4 \pm 7.0 \mathrm{mmHg}$. They explained that by anterior chamber inflammation and presence of inflammatory mediators, cytokines cause blood-ocular barrier breakdown detected in the eye for up to 1 month after intraocular surgery \& Lommatzsch et al. [20] reported that there was no significant change in the peripapillary RNFL thickness $(p=0.88)$ and the GCL complex $(p=0.97)$ their study included 19 eyes and a mean intraocular pressure (IOP) of $21.0 \mathrm{mmHg}$ with a significant reduction in IOP at each follow-up after lowering IOP by trabeculectomy with mitomycin $\mathrm{C}$ and follow up for 6 months $(\mathrm{p}=0.0001)$. This discrepancy than our results may be explained by their relatively low the starting IOP or by the magnitude of the IOP reduction. In this study, we analyzed Changes following IOP reduction that we found there was a non-significant increase in average RPCD, laminal optic nerve head vessel density (VD ID) \& whole image optic nerve vessel density thickness from baseline to 1, 3 months and significant increase in their vessel density from 3 months to 6 months (P value 0.04, 0.003, 0.01). Improvement of vessel density in our study has occurred may be due to wide range of IOP reduction ( $>50 \%$ ) that has relieved mechanical pressure over blood vessels. Also, we had about $80 \%$ of cases has treated with medical treatment and it is thought that antiglaucoma medications has a role in general vascular improvement of the eye $\&$ younger average age of our cases (42.60 years old) compared with older average age of some other papers that reflects on general vascular state of the patient and presence of general vascular diseases like hypertension, diabetes \& atherosclerosis that will reflect in return on their response to IOP reduction vascular effect on eye. Our results were the same like Hollò [21] who has studied on six eyes of 4 consecutive newly detected young male (32 and 45 years), reported an increase in peripapillary perfusion in 2 and 4 weeks after reducing the IOP medically. His study, differs from the others by a significantly higher baseline IOP (35-42 $\mathrm{mmHg}$ ) and more marked post treatment IOP reduction \& Alnawaiseh et al. [22] reported alteration of macular and papillary VD after iStent inject insertion in combination with phacoemulsification in patients with POAG $(n=24)$ compared with eyes having cataract surgery only $(n=24)$. Despite a relatively low baseline IOP of $18.2 \mathrm{mmHg}$ in the iStent group, the study demonstrated in the area of the ONH at the RPC layer, there was a significant improvement in VD with respect to the intrapapillary value (VD ID, $\mathrm{P}=0.012$ ) and the total papillary value ( $\mathrm{pVD}$ whole $\mathrm{P}=0.011$ ). However, the peripapillary total VD was not significantly changed (RPC: $43.5 \pm$ 7.7; after: $45.4 \pm 6.5, \mathrm{P}=0.421)$ ). Also, Shin et al. [23] have shown a significant increase in vessel density at the level of the lamina cribrosa 3 months after trabeculectomy which IOP significantly decreased from $26.3 \mathrm{~mm} \mathrm{Hg}$ to $12.5 \mathrm{~mm}$ $\mathrm{Hg}$, the microvascular improvement was observed in 19 eyes $(61.3 \%)$ of at total 31 POAG patients included in their study, although the RPCD was increased from $(44.9 \% \pm 6.0 \%$ to $47.0 \% \pm 7.2 \%)$ this difference was not statistically significant $(\mathrm{P}=0.133)$. On the other hand, de Paula et al. [17] in their study on 14 POAG patients with mean age $69.2 \pm 6.15$ years, the mean IOP was $23.2 \pm 3.21 \mathrm{mmHg}$ at baseline $\&$ a significant IOP reduction. They found that the RPC density remained relatively unchanged after the IOP reduction. It may be explained by older age of their patients \& lower starting IOP or by the magnitude of the IOP reduction from baseline; the superior-nasal sectors showed a greater capillary density com- 
pared with inferior-nasal one in these patients, Lommatzsch et al. [20] found no significant change in the whole papillary VD (pVD whole) as well as in the intrapapillary (VD ID) and peripapillary (RPCD) after successful trabeculectomy \& T. W. Ch'ng et al. [19] during the follow-up period, no association between changes in peripapillary VD and changes in IOP were found in their cohort, Peripapillary VD fluctuated widely throughout the follow up remaining under the mean baseline value. Also, Zeboulan et al. [24] did not find significant changes of peripapillary VD in their cases $(\mathrm{P}=0.788)$ they observed the absence of modification of vessel density after surgical IOP reduction, although some cases (6 patients, $28.5 \%$ ) showed an improvement in vessel density, this may indicate that IOP reduction itself does not work as a crucial factor in improving retinal microvasculature after surgical intervention, their mean baseline IOP was of $23.7 \pm 9.5$ $\mathrm{mmHg}$ and a mean IOP of $12.2 \pm 3.5$ $\mathrm{mmHg}$ ( $\geq 40 \%$ reduction) VD 1 month after deep non-penetrating sclerectomy in 21 glaucomatous eyes with mean age was $61.1 \pm 3.24$ years. In our study, there was a significant increase in foveal, parafoveal, perifoveal and whole macular vessel density from baseline to 1, 3 months and reached the best vascular improvement at 6 months post-treatment. The same found in $\mathrm{Al}$ nawaiseh et al.'s study [22], a significant improvement in VD remains in the superficial plexus over the entire macular area after combined cataract surgery and iStent implantation group (superficial OCT angiogram: before: 44.6 \pm 2.9 ; after: $47.6 \pm 4.5 ; \mathrm{P}=0$ 002; deep OCT angiogram: before: $50.9 \pm 3.6$; after: $53.0 \pm 4.2 ; \mathrm{P}=0$ 034), despite low baseline IOP. This postoperative increase in VD can be explained by that is the iStent group received intra-cameral acetylcholine to narrow the pupil for iStent implantation after phacoe-mulsification. Acetylcholine results in vasodilatation, so the increase in VD may have been due to pharmacology. However, Lommatzsch et al. [20] found that macular VD, neither in the superficial nor in the deep segmentation layer was any significant change in the VD evident at any postoperative time \& Ze'boulon et al. [24] found no significant change in in the macular region 1 month post filtering surgery but there was a significant change only in the inferotemporal area of patients with predominantly superior visual field defects $(\mathrm{p}=0.024)$, this discrepancy of their results from ours may be referred again to older age group of their patients and lower IOP in baseline with lower range of IOP reduction. Our study has some limitations, relatively small sample size \& the follow-up was a relatively shortterm \& careless of patients about their health state problems and follow up appointments. Our study clearly emphasizes the role of OCT-A as a diagnostic and prognostic tool in the management and follow up of glaucoma patients. OCT-A substantiates the vascular dysregulation theory in the pathogenesis of glaucoma.

\section{Conclusion}

In our study, a significant increase in peripapillary capillary density "superficial and deep vascularity" at sixth month of follow up and significant increase of macular vessel density from first month of follow up and increased with third and six months with IOP reduction may be related to wide range of IOP reduction that has relieved mechanical pressure over blood vessels and medical drugs of eye drops thought to have a role in improvement of vascularity. OCTA is a very valuable tool to document the macular and $\mathrm{ONH}$ capillary density changes in early glaucoma diagnosis and follow up. We recommend future studies should focus on comparing the medical and surgical way of treatment and their effect on retinal vascularity as a reflection for IOP reduction 


\section{References}

1. Tham, Y-C., Li, X., Wong, T., et al. Global prevalence of glaucoma and projections of glaucoma burden through 2040. Ophthalmology. 2014; 121: 2081-2090.

2. European Glaucoma Society Terminology and Guidelines for Glaucoma, $4^{\text {th }}$ ed., Ch 3: Treatment principles and options supported by the EGS Foundation. Br. J. Ophthalmol. 2017; 101: 130-195.

3. Mansouri, K. Optical coherence tomography angiography and glaucoma: Searching for the missing link. Expert Rev Med Devices. 2016; 13: 879880.

4. Michelessi, M., Lucenteforte, E., Oddone, F., et al. Optic nerve head and fibre layer imaging for diagnosing glaucoma. Cochrane Database Syst Rev. 2015; p CD008803.

5. Plange, N., Kaup, M., Weber, A., et al. Fluorescein filling defects and quantitative morphologic analysis of the optic nerve head in glaucoma. Arch Ophthalmol. 2004; 122: 195-201.

6. O'Brart, D., de Souza Lima, M., Bartsch, D., et al. Indocyanine green angiography of the peripapillary region in glaucomatous eyes by confocal scanning laser ophthalmoscopy. Am. J. Ophthalmol. 1997; 123: 657-666.

7. Rechtman, E., Harris, A., Kumar, R., et al. An update on retinal circulation assessment technologies. Curr Eye Res. 2003; 27: 329-343.

8. Nicolela, M., Hnik, P., Drance, S. Scanning laser Doppler flowmeter study of retinal and optic disk blood flow in glaucomatous patients. Am. J. Opht-halmol. 1996; 122: 775-783.

9. Yaoeda, K., Shirakashi, M., Funaki, S., et al. Measurement of microcirculation in the optic nerve head by laser speckle flowgraphy and scanning laser Doppler flowmetry. Am. J. Ophthalmol. 2000; 129: 734-739.
10. Shoji, T., Zangwill, L., Akagi, T., et al. Progressive macula vessel density loss in primary open-angle glaucoma: A longitudinal study. Am. J. Ophthalmol. 2017; 182: 107=117.

11. Lee, E., Lee, K., Lee, S., et al., OCT angiography of the peripapillary retina in primary open-angle glaucoma. Invest Opthalmol Vis Sci. 2016; 57: 6265-6270.

12. Grunwald, J., Sinclair, S., Riva, C. Autoregulation of the retinal circulation in response to decrease of intraocular pressure below normal. Invest Ophthalmol Vis Sci. 1982; 23: 124-127.

13. Flammer, J., Orgul, S. Optic nerve blood flow abnormalities in glaucoma. Prog Retin Eye Res. 1998; 17 (2): 267-289.

14. Liu, L., Jia, Y., Takusagawa, H., et al. Optical coherence tomography angiography of the Peripapillary Retina in Glaucoma. JAMA Ophthalmol. 2015; 2133 (9): 1045-1052

15. Xu, H., Kong, X. Study of retinal microvascular perfusion alteration and structural damage at macular region in primary open angle glaucoma patients. Zhonghua Yan Ke Za Zhi Chin J. Ophthalmol 2017; 53: 98-103.

16. Jia, Y., Morrison, J., Tokayer, J., et al. Quantitative OCT angiography of optic nerve head blood flow. Biomed Opt Express. 2012; 3: 3127-3137.

17. De Paula, A., Perdicchi, A., Di Tizio, F., et al. Effect of intraocular pressure lowering on the capillary density of optic nerve head and retinal nerve fiber layer in patients with glaucoma. European J. of Ophthalmology. 2020; 31 (6): 3003-3009

18. Sarkar, K., Das, P., Pal, R., et al. Optical coherence tomographic assessment of retinal nerve fiber layer thickness changes before and after glaucoma filtration surgery. Oman $\mathbf{J}$ Ophthalmol. 2014; 7 (1): 3-8. 
19. Ch'ng, T., Gillmann, K., Hoskens, K., et al. Effect of surgical intraocular pressure lowering on retinal structures - nerve fibre layer, foveal avascular zone, peripapillary and macular vessel density: 1-year results. Eye. 2020; 34: 562-571.

20. Lommatzsch, C., Rothaus, K., Koch, J., et al. Retinal perfusion 6 months after trabeculectomy as measured by optical coherence tomography angiography. Int Ophthalmol. 2019; 39 (11): 2583-2594

21. Hollo, G. Influence of large intraocular pressure reduction on peripapillary OCT vessel density in ocular hypertensive and glaucoma eyes eyes. $\boldsymbol{J}$. Glaucoma. 2017; 26: e7-e10.

22. Alnawaiseh, M., Müller. V., Lahme, L., et al. Changes in flow density measured using optical coherence tomography angiography after istent insertion in combination with phac-oemulsification in patients with open-angle glaucoma. J. Ophthalmol. 2018: 2890357.

23. Shin, J., Sung, K., Uhm, K., et al. Peripapillary microvascular improvement and lamina cribrosa depth reduction after trabeculectomy in primary open-angle glaucoma. Invest Ophthalmol Vis Sci. 2017; 58: 59935999.

24. Zéboulon P, Le've^que P-M, Brasnu $E$ et al Effect of surgical intraocular pressure lowering on peripapillary and macular vessel density in glaucoma patients: an optical coherence tomography angiography study. J. Glaucoma. 2017; 26: 466-472. 